\title{
Osteocalcin regulates murine and human fertility through a pancreas-bone-testis axis
}

\author{
Franck Oury, ${ }^{1}$ Mathieu Ferron, ${ }^{1,2}$ Wang Huizhen, ${ }^{3}$ Cyrille Confavreux, ${ }^{4}$ Lin Xu, ${ }^{1}$ Julie Lacombe, ${ }^{5}$ \\ Prashanth Srinivas, ${ }^{1}$ Alexandre Chamouni, ${ }^{1}$ Francesca Lugani, ${ }^{6}$ Herve Lejeune, ${ }^{7}$ \\ T. Rajendra Kumar, ${ }^{3}$ Ingrid Plotton, ${ }^{8}$ and Gerard Karsenty ${ }^{1}$
}

\begin{abstract}
1Department of Genetics and Development, Columbia University, New York, New York, USA. ${ }^{2}$ nnstitut de Recherches Cliniques de Montreal, Montreal, Quebec, Canada. ${ }^{3}$ Department of Molecular and Integrative Physiology, University of Kansas Medical Center, Kansas City, Kansas, USA. ${ }^{4}$ INSERM U1033, Université de Lyon, Department of Rheumatology, Hôpital Edouard Herriot, Hospices Civils de Lyon, Lyon, France. ${ }^{5}$ Department of Cell Biology and Ruth L. and David S. Gottesman Institute for Stem Cell and Regenerative Medicine Research, Albert Einstein College of Medicine, New York, New York, USA. ${ }^{6}$ Department of Medicine, Columbia University, New York, New York, USA. ${ }^{7}$ Service de Médecine de la Reproduction, Hôpital Femme-Mère-Enfant, CHU de Lyon, Bron, France. ${ }^{8}$ Service d'Hormonologie Endocrinologie Moléculaire, Groupement Hospitalier Est Hospices Civils de Lyon, Lyon, France.
\end{abstract}

\begin{abstract}
The osteoblast-derived hormone osteocalcin promotes testosterone biosynthesis in the mouse testis by binding to GPRC6A in Leydig cells. Interestingly, Osteocalcin-deficient mice exhibit increased levels of luteinizing hormone (LH), a pituitary hormone that regulates sex steroid synthesis in the testes. These observations raise the question of whether LH regulates osteocalcin's reproductive effects. Additionally, there is growing evidence that osteocalcin levels are a reliable marker of insulin secretion and sensitivity and circulating levels of testosterone in humans, but the endocrine function of osteocalcin is unclear. Using mouse models, we found that osteocalcin and LH act in 2 parallel pathways and that osteocalcin-stimulated testosterone synthesis is positively regulated by bone resorption and insulin signaling in osteoblasts. To determine the importance of osteocalcin in humans, we analyzed a cohort of patients with primary testicular failure and identified 2 individuals harboring the same heterozygous missense variant in one of the transmembrane domains of GPRC6A, which prevented the receptor from localizing to the cell membrane. This study uncovers the existence of a second endocrine axis that is necessary for optimal male fertility in the mouse and suggests that osteocalcin modulates reproductive function in humans.
\end{abstract}

\section{Introduction}

Osteocalcin is an osteoblast-specific secreted protein that, when undercarboxylated, is a multifunctional hormone. It acts on pancreatic $\beta$ cells to increase insulin secretion, on muscle and white adipose tissue to promote glucose homeostasis, and on Leydig cells of the testis to favor testosterone biosynthesis following its binding to a specific receptor, GPRC6A (1-6). The biological relevance of its latter function is evidenced by the fact that male mice lacking Osteocalcin or this receptor specifically in Leydig cells demonstrate a marked reduction of circulating testosterone levels, a decrease in sperm count, and mediocre fertility $(2,4)$. These findings raise a series of questions. The answers to these questions are needed to establish the biological importance of this function of bone.

For instance, a remarkable feature of the reproduction phenotype observed in Osteocalcin-null (Osteocalcin ${ }^{-/}$) mice is that it develops in the face of an increase in circulating levels of luteinizing hormone (LH), a pituitary hormone that is the main regulator of sex steroid synthesis by Leydig cells (7-9). This situation raises the following question: Does osteocalcin act downstream of $\mathrm{LH}$ or does this feature reveal the existence of 2 different pathways, one pituitary dependent and one bone dependent, that would both be necessary for male fertility? If the latter case is true, then the next question is this: how is the reproductive function of osteocalcin regulated?

A second important issue raised by the demonstration that osteocalcin is a hormone in the mouse is whether that is also the case in humans. While there is a growing body of evidence that osteocalcin serum levels are a reliable indicator of the degree of

Conflict of interest: The authors have declared that no conflict of interest exists. Citation for this article: J Clin Invest. 2013;123(6):2421-2433. doi:10.1172/JCI65952. insulin secretion, insulin sensitivity, and circulating serum testosterone levels in humans (10-13), there is, for now, no genetic evidence establishing that osteocalcin fulfills its endocrine functions in humans. The identification of a functional osteocalcin receptor, and the realization that osteocalcin influences male fertility provide an opportunity to address this issue by searching for mutations in either Osteocalcin or GPRC6A in a well-defined pathological context, namely in patients with peripheral testicular failure of unknown origin.

In addressing these 2 issues, we first provide evidence obtained through cell-specific gene deletion experiments in the mouse that support the existence of a pancreas-bone-testis axis favoring male fertility acting in parallel to and independently of the hypothalamus-pituitary-testis axis. In trying to expand the biological relevance of osteocalcin from mouse to human, we identified in 2 patients with peripheral testicular failure the same amino acid substitution affecting a highly conserved residue in 1 of the 7 transmembrane domains of GPRC6A. This mutation prevents this receptor from reaching the cell membrane and acts in a dominant negative manner in cell culture and in vivo. Together, these results shed new light on the regulation of male fertility in vertebrates.

\section{Results}

Osteocalcin and LH define 2 modes of regulating male fertility in the mouse. The main endocrine pathway regulating male fertility is the hypothalamo-pituitary axis, in which $\mathrm{LH}$, a heterodimer between an $\alpha$-subunit common to several peptide hormones and a $\beta$-subunit specific to $\mathrm{LH}$, favors testosterone biosynthesis $(7,14,15)$. Although less severe, the reproductive phenotype of Osteocalcin ${ }^{-}$ and Gprc6a-/- male mice bears some resemblance to the one seen 

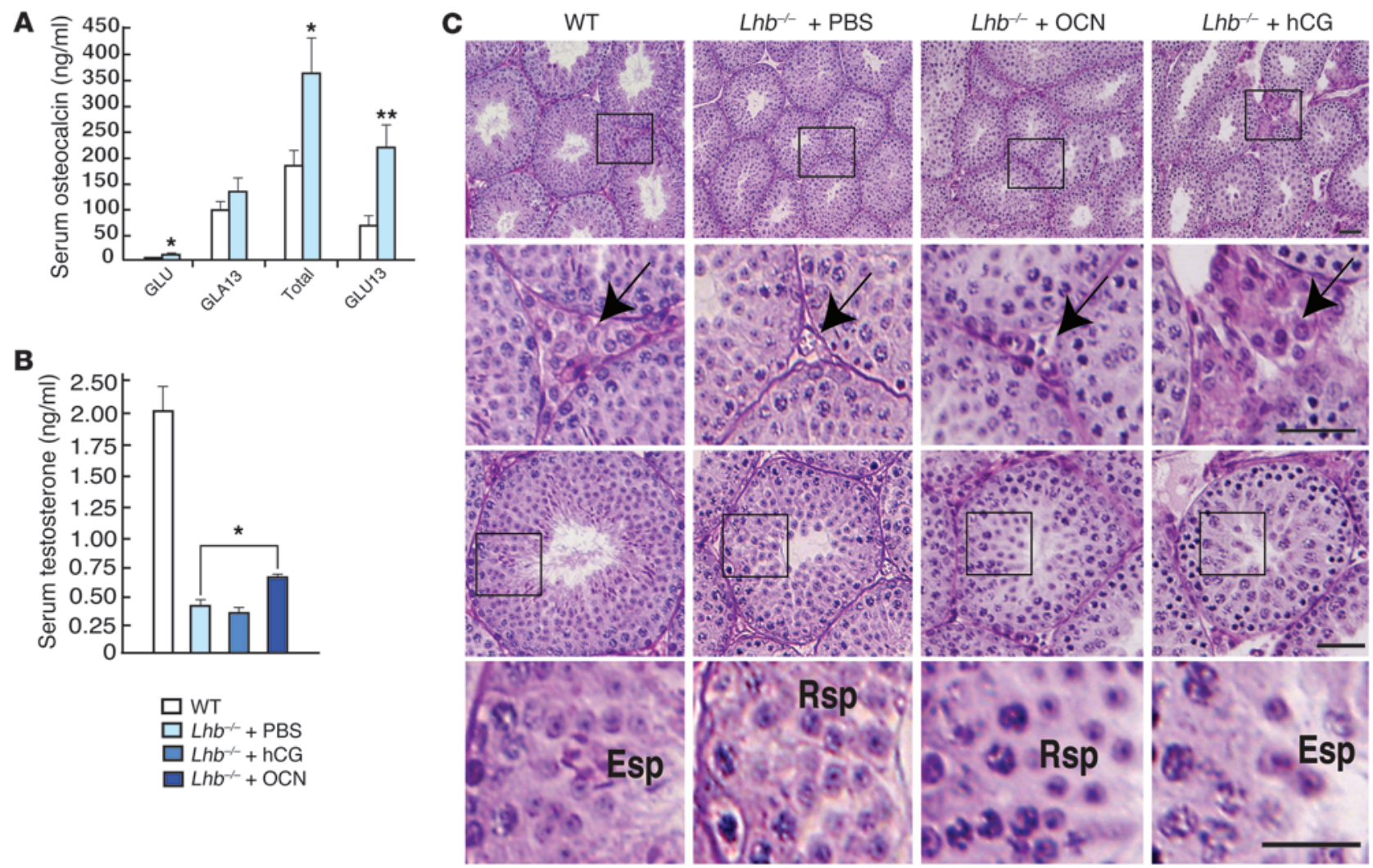

D
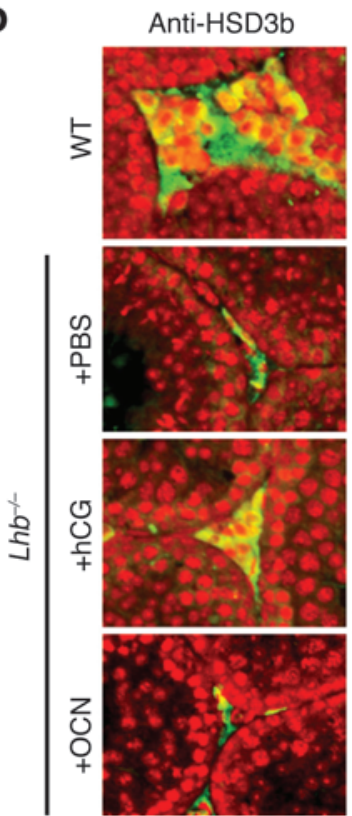

Anti-Cyp11a
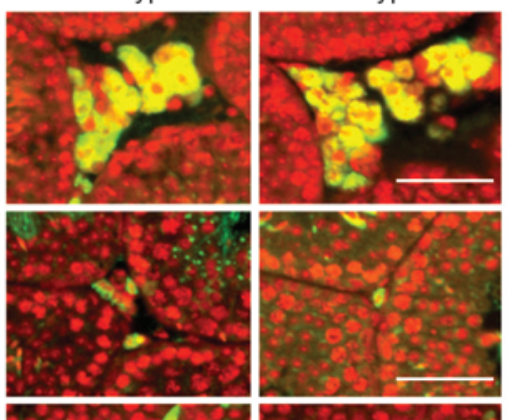
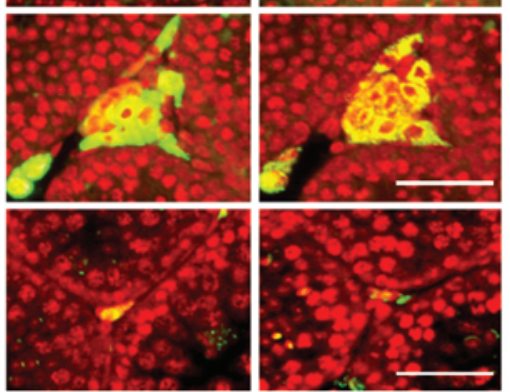

E

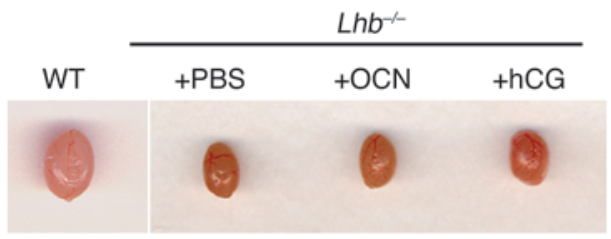

$\mathbf{F}$

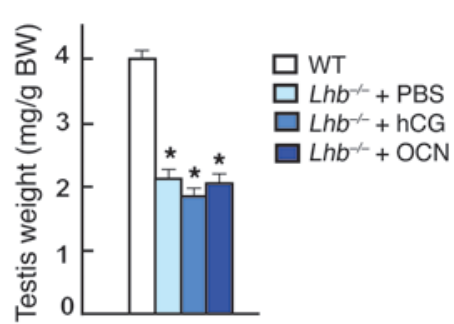

Figure 1

Analysis of the rescue of male fertility phenotype in $L h b^{-/}$male mice after osteocalcin injections. (A) Measurement of the uncarboxylated (GLUOCN), carboxylated (GLA13-OCN), total (total-OCN), and undercarboxylated (GLU13-OCN) forms of osteocalcin in the serum of 10-week-old Lh-deficient mice $\left(L h b^{-/}\right)(n=7)$ versus WT $(n=5)$. (B) Circulating testosterone levels in 12-week-old WT $(n=5)$ and $L h b^{-/-}(n=7)$ mice and in Lhb $b^{-1-}$ mice injected for 1 month with PBS $(n=8)$ or osteocalcin (3 ng/ml) $(n=5)$. (C and D) Testes cross-sections of WT and Lhb ${ }^{-/-}$injected for 1 month with PBS, osteocalcin (3 ng/g/d) or hCG (5 UI twice a week). (C) Histological demonstration of the interstitial tissue hypoplasia (2 first rows) and of the absence of spermatogenesis (2 last rows) observed in Lhb $b^{-/}$mice. While injections of hCG rescue the phenotype, PBS or osteocalcin do not. The black line delimits the frame zoom shown in the second row. The head arrows point to the interstitial tissue containing Leydig cells. Rsp, round spermatids; Esp, elongated spermatids. (D) Immunofluorescence, using anti-Cyp17, anti-3 $\beta$-HSD, or anti-Cyp11a antibodies as markers of mature Leydig cells. (E) Testis size and (F) testis weight normalized to BW (mg/g of BW) in Lhb $b^{-/}$mice injected with PBS $(n=8)$, osteocalcin $(n=4)$, or hCG $(n=3)$ compared with WT mice. All analyses were performed on nonbreeder C57BL/6J mice. Scale bar: $100 \mu$ m. ${ }^{*}<0.05$; ${ }^{\star \star} P<0.01$. 
A

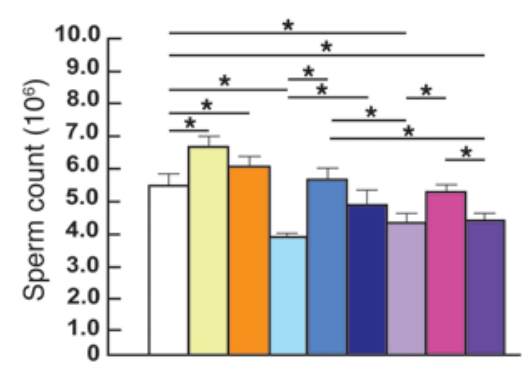

C
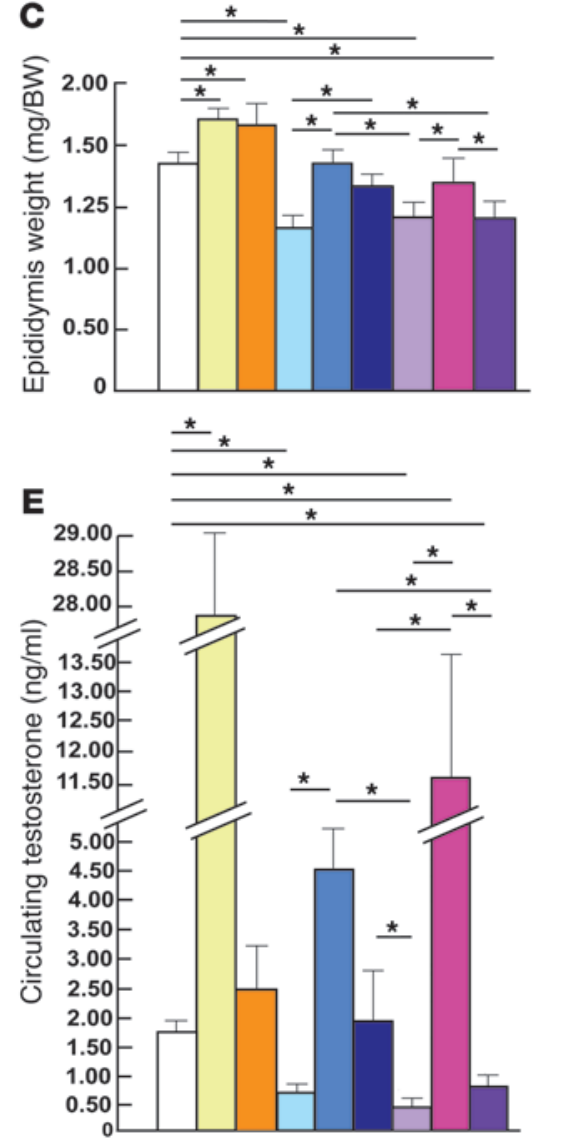

B

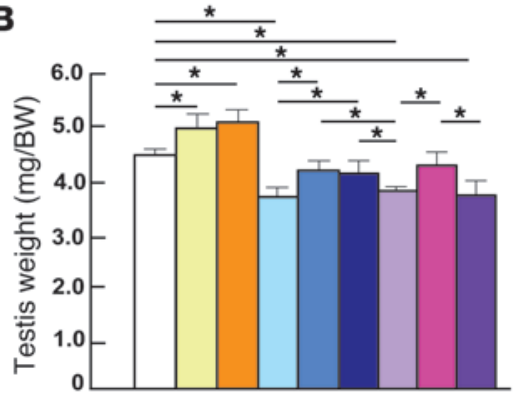

D

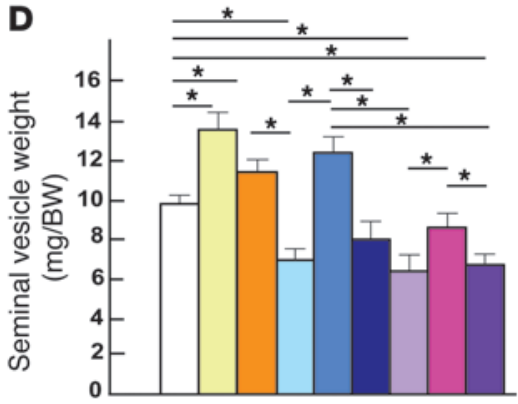

\section{Figure 2}

Analysis of Osteocalcin-/- male mouse fertility after hCG or osteocalcin injection. Sperm count (A); testis (B), epididymal (C), and seminal vesicle (D) weights normalized to BW ( $\mathrm{mg} / \mathrm{g}$ of BW), (E) circulating testosterone levels in WT and Osteo$\mathrm{calcin}^{-1}$ mice at 10 weeks of age, both injected either with PBS (WT, $n=20$; Osteocalcin ${ }^{-1-} ; n=14$; Gprc6a- ${ }^{-/}, n=7$ ), hCG (WT, $n=12$; Osteocalcin $^{-1}, n=8$; Gprc6a $\left.^{-1}, n=6\right)$ (5 UI, twice a week), or osteocalcin (WT, $n=6$; Osteocalcin ${ }^{-1-}$, $n=6$; $\left.\operatorname{Grc6a}^{-1-}, n=6\right)(3 \mathrm{ng} / \mathrm{g} / \mathrm{d})$. All analyses were performed on nonbreeder 129/Sv mice. Error bars represent SEM. ${ }^{*} P<0.05$, Student's t test. Specifically, $P<0.05$ versus WT injected with hCG ( $5 \mathrm{UI}$, twice a week); and $P<0.05$ versus WT injected with osteocalcin $(3 \mathrm{ng} / \mathrm{g} / \mathrm{d})$. in $\mathrm{Lbb}^{-/-}$(LH-deficient) male mice, as they are both characterized by a defect in testosterone synthesis and testosterone-dependent events $(2,3,7)$. However, LH circulating levels are high in Osteocal$\mathrm{cin}^{-/-}$male mice. These observations prompted us to ask whether $\mathrm{LH}$ may regulate osteocalcin reproductive function.

If this were the case, one would expect that circulating levels of the undercarboxylated (active) form of osteocalcin should be low in $\mathrm{Lhb}^{-/-}$male mice and that injections of osteocalcin would rescue, completely or at least in part, the hypotestosteronemia of $\mathrm{Lhb}^{-1-}$ male mice. However, circulating levels of undercarboxylated (GLU13) osteocalcin were not lower in $\mathrm{Lbb}^{-/-}$than in WT male mice (Figure 1A), and daily injections of osteocalcin for 1 month in 6-week-old $\mathrm{Lhb}^{-/-}$male mice did not normalize circulating testosterone levels (Figure 1B). Moreover, histological analysis of testes of 10-week-old $\mathrm{Lhb}^{-/-}$male mice injected with osteocalcin failed to show any improvement in spermatogenesis or a reversal of their Leydig cell hypoplasia (Figure 1, C and D, and Supplemental Figure 1A; supplemental material available online with this article; doi:10.1172/JCI65952DS1). The dose of osteocalcin used in this experiment was chosen because it is sufficient to increase testosterone synthesis by Leydig cells in culture (2). Immunofluorescence analysis of mature Leydig cells using anti-Cyp17, anti-3 $\beta$-HSD or anti-Cyp11a antibodies showed that daily injections of osteocalcin for 30 days did not increase the number of Leydig cells in $\mathrm{Lhb}^{-/-}$male mice, while injections of the placental homolog of $\mathrm{LH}$, human chorionic gonadotropin (hCG), did (Figure 1D and Supplemental Figure 1A). Therefore, and unlike what was achieved by hCG injections, osteocalcin injections did not increase testis size and weight, Leydig cell count, or spermatogenesis in $\mathrm{Lbb}^{-1-}$ male mice (Figure 1, $\mathrm{E}$ and $\mathrm{F}$ ). This was not due to a poor bioactivity of the recombinant protein, since the same preparation of osteocalcin corrected all reproductive abnormalities seen in Osteocalcin ${ }^{-/-}$mice but not in Gprc6a-/- mice (Figure 2). To rule out that the failure 
Fetal

A liver HSC

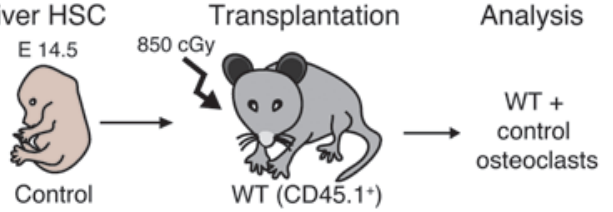

Control

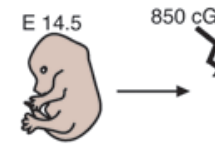

Ctsk-cre;DTA ${ }^{n+*}$

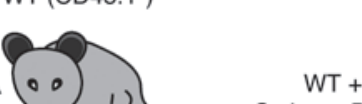

WT +

Ctsk-cre;DTA
osteoclasts

WT (CD45.1+)
B

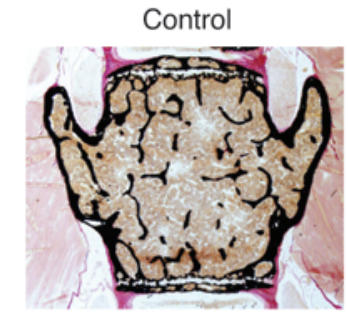

BV/TV (\%) $\quad 12.9 \pm 0.3$

C

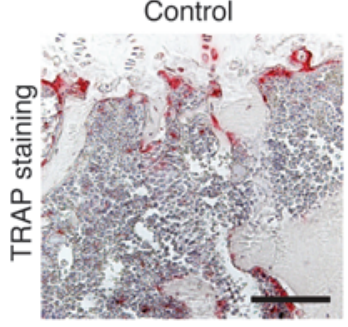

Oc.S/BS (\%) $9.53 \pm 0.95$

N.Oc./PM $(1 / \mathrm{mm}) \quad 0.31 \pm 0.03$

D

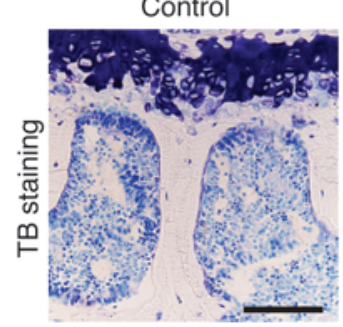

Ctsk-cre;DTA $A^{f l+}$

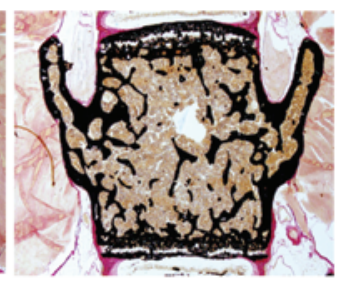

$21.4 \pm 0.7 * \star \star *$

Ctsk-cre;DTA $A^{f /+}$

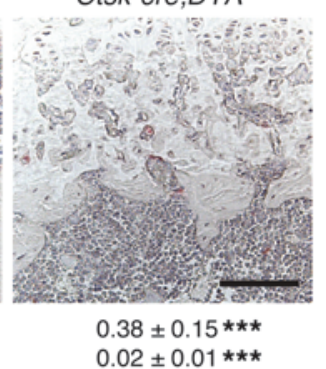

Ctsk-cre;DTA ${ }^{n /+}$

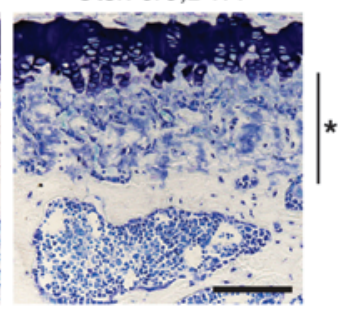

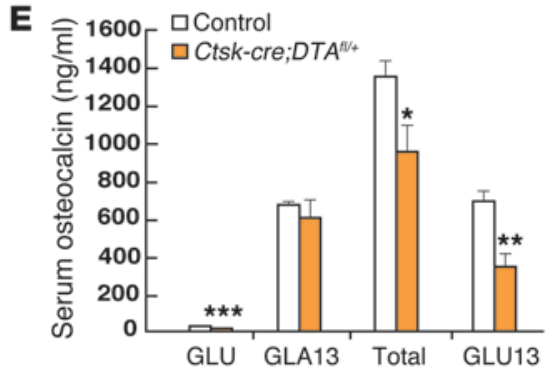

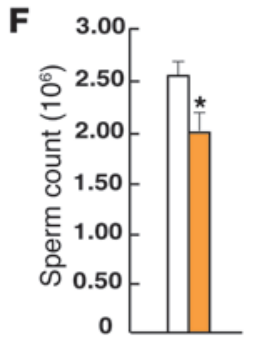

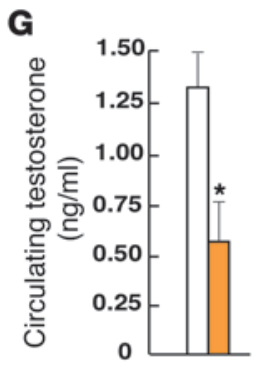
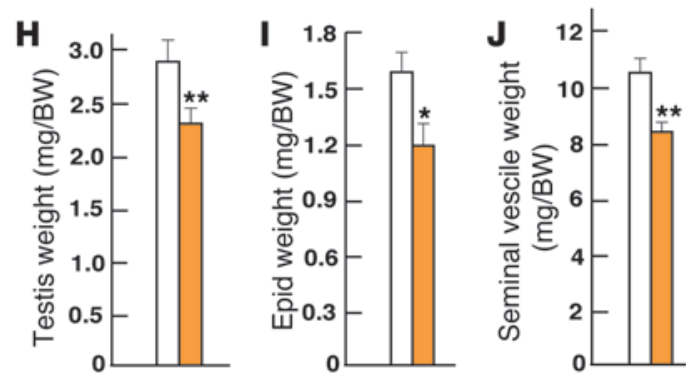

K

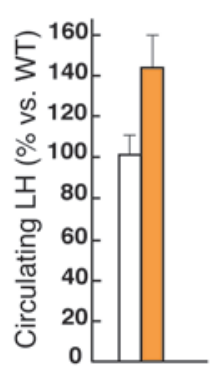

L

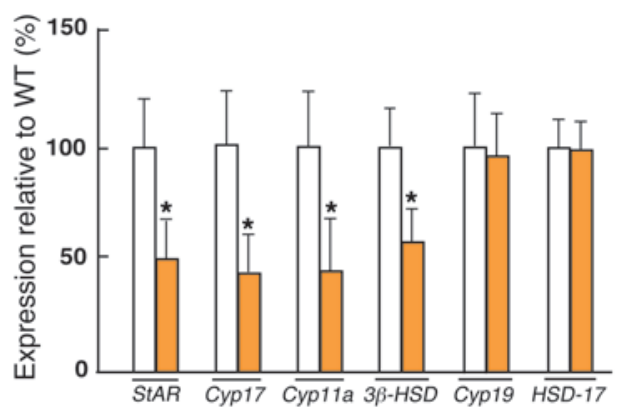

Figure 3

The osteocalcin reproductive function is hampered in the absence of proper bone resorption. (A) Schematic representation of the strategy used to generate Ctsk-Cre;DTA ${ }^{\mathrm{fl}+}$ male mice. (B-D) Histological and histomorphometric analyses of vertebrae in control $(n=8)$ and Ctsk-Cre;DTA $A^{f / /+}$ $(n=9)$ male mice 4 months after transplantation. (B) Von Kossa/Van Giesen staining. Bone volume over trabecular volume (BV/TV\%) is indicated below the pictures. (C) TRAP staining to reveal osteoclasts. Osteoclast surface per bone surface (Oc.S/BS [\%]) and number of osteoclasts per bone trabecular surface (N. Oc/Bpm [1 mm]) are indicated below the pictures. (D) Toluidine blue staining showing an important presence of cartilage remnants (indicated by the asterisk) in Ctsk-Cre;DTA fl/ male mice versus WT. (E) Measurement of uncarboxylated, carboxylated, total, and undercarboxylated forms of osteocalcin in the serum of 10-week-old Ctsk-Cre;DTA ${ }^{\mathrm{fl}+}$ versus WT male mice. (F) Sperm counts and $(\mathbf{G})$ circulating testosterone levels; $(\mathbf{H}-\mathbf{J})$ testis, epididymal, and seminal vesicle weights normalized to BW $\left(\mathrm{mg} / \mathrm{g}\right.$ of BW) in Ctsk-Cre;DTA ${ }^{\mathrm{fll}+}(n=12)$ versus WT $(n=11)$ male mice. (K) Circulating LH measurement in control and Ctsk-Cre;DTA ${ }^{f l+}+$ mice. (L) qPCR analysis of the expression of steroidogenic acute regulatory protein (StAR), cholesterol side-chain cleavage enzyme (Cyp11a), cytochrome P-450 $17 \alpha$ (Cyp17), 3- $\beta$-hydroxysteroid dehydro-

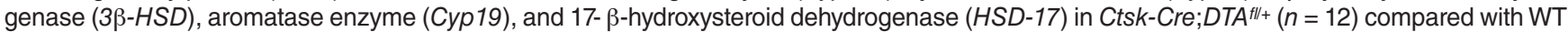
$(n=11)$ male mice. All analyses presented were performed on nonbreeder C57BL/6J mice. Scale bars: $200 \mu \mathrm{m}) .{ }^{*} P<0.05 ;{ }^{* \star} P<0.01 ;{ }^{* \star \star} P<0.001$.

of the osteocalcin injections to increase testis weight was due to the fact that they were performed in adult mice, we repeated this experiment in 10-day-old $\mathrm{Lhb}^{-/-}$mice that were injected for 30 days with either hCG (5 UI, twice a week) or osteocalcin ( $3 \mathrm{ng} / \mathrm{g}$ of BW, daily). Here again, while hCG treatment increased Leydig cell number, osteocalcin did not (Supplemental Figure 1B).

To further distinguish between LH-dependent and osteocalcindependent regulation of male fertility, we performed the reverse 
A

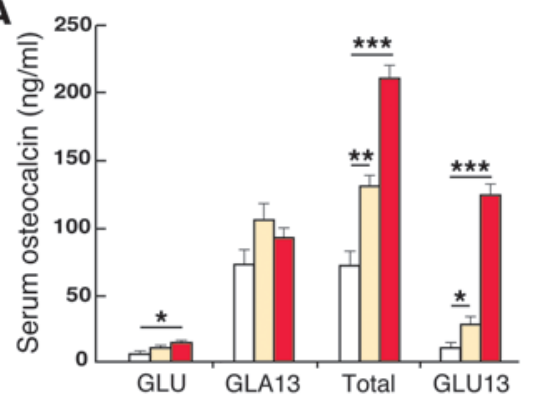

F

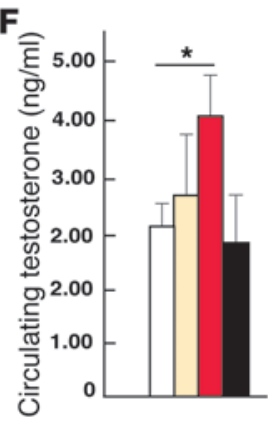

B

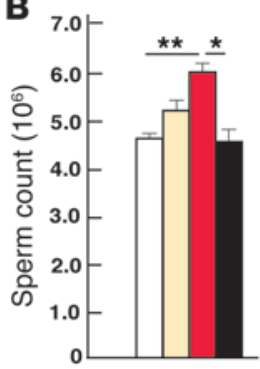

C

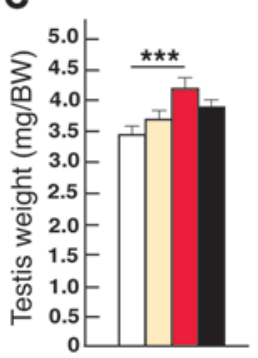

D

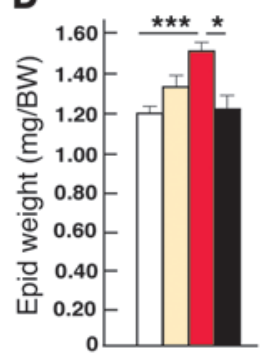

E

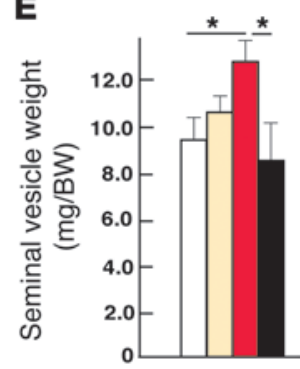

G

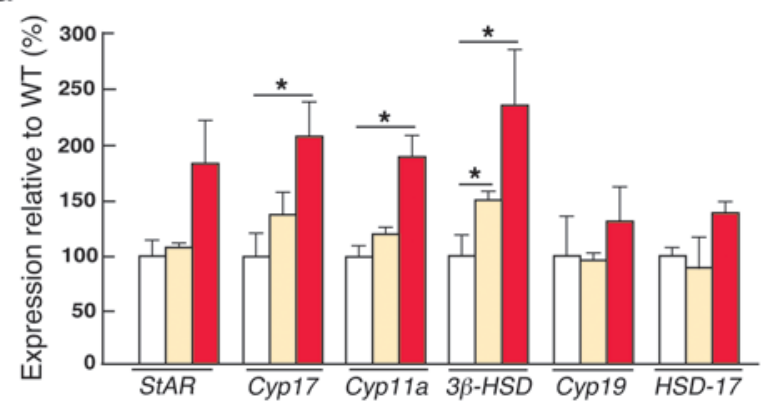

H

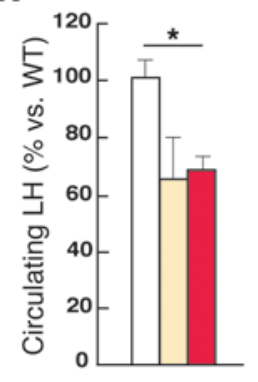

口WT

$\square \mathrm{Opg}^{+/}$

- $\mathrm{Opg}^{\prime-}$

- Opg ${ }^{+/}$; Osteocalcin ${ }^{+/}$

Figure 4

An increase in osteoclast number favors both osteocalcin activity and male fertility. (A) Measurement of the uncarboxylated, carboxylated, total, and undercarboxylated forms of osteocalcin in serum of WT $(n=12), \mathrm{Opg}^{-/-}(n=10)$, and $\mathrm{Opg}^{+/-}$mice $(n=10)$. (B) Sperm count; (C) testis, (D) epididymal; and (E) seminal vesicle weights normalized to BW (mg/g of BW). (F) Circulating testosterone levels in WT $(n=12)$, Opg ${ }^{-/}$ $(n=10), \mathrm{Opg}^{+/-}(n=10)$, and Osteocalcin ${ }^{+-} ; \mathrm{Opg}^{+/-}(n=6)$ mice at 12 weeks of age. (G) qPCR analysis of the expression of StAR, Cyp11a, Cyp17, 3ß-HSD, Cyp19, and HSD-17 in Opg-/- $(n=10)$ and $\mathrm{Opg}^{+/-}(n=10)$ compared with WT $(n=12)$ male mice. (H) Circulating LH measurement in $\mathrm{Opg}^{-/-}(n=10)$ and $\mathrm{Opg}^{+-}(n=10)$ compared $(\%)$ with WT $(n=12)$ male mice. All analyses presented were performed on nonbreeder C57BL/6J mice. ${ }^{*} P<0.05 ;{ }^{* *} P<0.01 ;{ }^{* * *} P<0.001$.

experiment. Namely, we injected 6-week-old Osteocalcin ${ }^{-/}$or Gprc6a $a^{-/}$ male mice with hCG (5UI, twice a week) for 1 month, reasoning that if osteocalcin were a mediator of $\mathrm{LH}$ reproductive function, these injections would not improve the reproductive abnormalities seen in Osteocalcin ${ }^{-/-}$mice. Instead, this treatment normalized all parameters analyzed: testes, epididymal, and seminal vesicle weights, sperm count, and testosterone circulating levels in Osteocalcin ${ }^{-/-}$male mice. Finally, circulating LH level was equally decreased in WT and Osteocalcin $^{-/-}$serum after treatment with hCG (Supplemental Figure 1C). Taken together, these experiments suggest that $\mathrm{LH}$ promotes testosterone biosynthesis in an osteocalcin-independent manner.

Osteocalcin and $L H$ do not regulate each other. In the next set of experiments, we asked whether osteocalcin regulates $L h$ expression and/ or whether LH regulates Osteocalcin expression. For Osteocalcin to regulate $L h$ expression it would first require that its receptor, Gprc6a, is expressed in the hypothalamus or the pituitary gland.

To address this issue, we first studied the Gprc6a endogenous pattern of expression of by in situ hybridization. While expression of this gene could be detected in Leydig cells, it was undetectable in the hypothalamus and pituitary gland. Concerned that this technique was not sensitive enough, we then used a second and more sensitive assay, quantitative PCR (qPCR), to measure Gprc6a expression. As an internal negative control of specificity, we also used in this experiment tissues originating from $G p r c 6 a^{-/-}$mice. This assay failed to detect any expression of Gprc6a in the hypothalamus and pituitary gland in WT mice above what was seen in Gprc6a $a^{-/-}$mice (Supplemental Figure 2, A and B). We verified that the primers used could detect Gprc6a expression in WT but not
Gprc6a $\mathrm{a}^{-/-}$testes (Supplemental Figure 2B). Taken together, these 2 different experiments indicate that the regulation of testosterone synthesis by osteocalcin in Leydig cells cannot be ascribed to a measurable influence of osteocalcin signaling through Gprc6a in the hypothalamus or the pituitary gland.

Conceivably, however, LH could be required for osteocalcin stimulation of testosterone biosynthesis by Leydig cells. Evidence from 2 experiments suggested that this is not the case. First, the positive effect of osteocalcin on testosterone synthesis in Leydig cells was recorded when cells were maintained in serum-free medium, i.e., in the total absence of LH (2). Second and more directly, in cell culture, hCG does not regulate expression of Osteocalcin or of genes modifying it in osteoblasts (Supplemental Figure 3, A-C). Hence, taken collectively, results presented in Figures 1 and 2 suggest the notion that osteocalcin regulates male fertility independently of the hypothalamo-pituitary-axis; they also failed to provide any evidence that LH regulates Osteocalcin expression.

Bone resorption as a determinant of osteocalcin reproductive function in the mouse. By dissociating pituitary-dependent from bone-dependent regulation of male fertility, the experiments presented above raised the question of the identity of upstream regulators of osteocalcin reproductive function.

That the ability of osteocalcin to favor glucose homeostasis is determined by osteoclastic bone resorption prompted us to ask whether male fertility was another physiological function to be added to the credit of bone resorption (16). To address this question, we relied on 2 mouse models, a loss-of-function model and a gain-of-function model. 

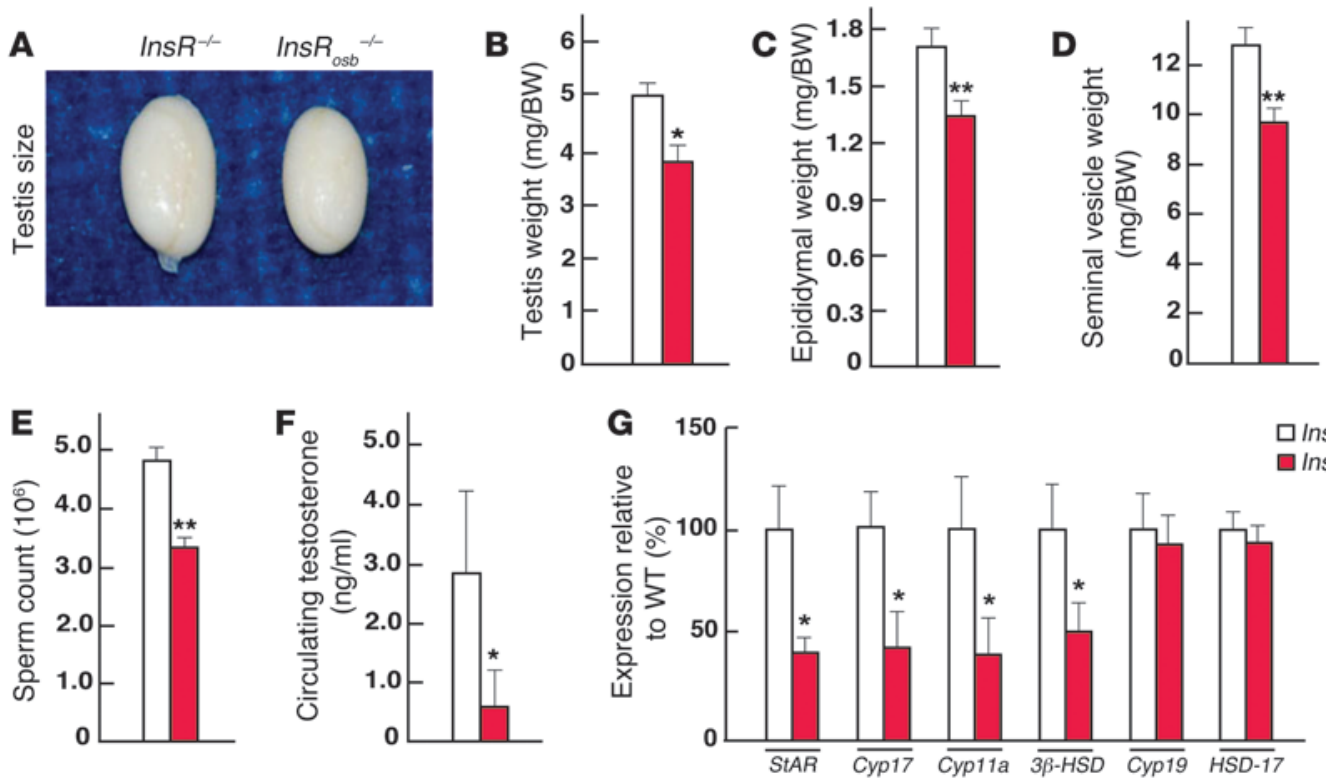

\section{Figure 5}

Insulin signaling in osteoblasts favors testosterone production. (A) Testis size, and (B) testis, (C) epididymal, and (D) seminal vesicle weights normalized to BW (mg/g of BW); (E) sperm count; (F) circulating testosterone levels in Ins $R_{o s b^{-/}}$versus WT male mice. (G) qPCR analysis of the expression of StAR, Cyp11a, Cyp17, 33-HSD, Cyp19, and HSD-17 genes in testes of $I n s R_{o s b^{-1-}}(n=10)$ and WT $(n=12)$ mice. All analyses presented were performed on nonbreeder mix background (129/Sv: 87.5\%; 129/Sv: $12.5 \%$ ) mice. ${ }^{\star} P<0.05 ;{ }^{* \star} P<0.01$.

First, we generated a mouse model demonstrating a profound depletion of the mature osteoclasts population by crossing Ctsk-Cre mice, in which the Cre recombinase is expressed in mature osteoclasts only (17), with $D T A^{f l /+}$ mice that express a flox-stop-flox diphtheria toxin subunit $\alpha$ gene (DTA) cassette under the control of the Rosa26 locus (18). DTA ${ }^{f /+}$ mice are phenotypically WT because the flox-stop-flox cassette present in front of the DTA gene prevents expression of the DTA protein in any tissues. However, Cre-mediated removal of the stop cassette in osteoclasts only, in Ctsk-Cre;DTA $A^{f /+}$ double knockin mice, leads to osteoclast-specific expression of DTA, a toxic protein, and osteoclast death (Figure 3A).

Ctsk-Cre;DTA ${ }^{f l /+}$ mice developed a classical osteopetrosis phenotype characterized by very dense bones, as seen in x-rays, and the absence of incisor eruption because of a severe impairment in bone resorption (Supplemental Figure 4A). All mutant animals died between 2 and 3 weeks of age. To circumvent this postnatal lethality, we transplanted Ctsk-Cre;DTA ${ }^{f l+}$ fetal liver HSCs into WT irradiated adult mice; since osteoclasts are of hematopoietic origin, mice transplanted with Ctsk-Cre;DTA ${ }^{f l+}$ HSCs should be depleted of osteoclasts. Four months after transplantation, bone and fertility phenotypes of these animals were analyzed.

Bone histomorphometry verified that Ctsk-Cre;DTA ${ }^{f l /+}$ transplanted mice developed a high bone-mass phenotype due to a 15 -fold decrease in the number of osteoclasts (Figure 3, B and C). The presence of cartilage remnants characterized this osteopetrosis phenotype (Figure 3D). These osteopetrotic mice showed more than a 2 -fold decrease in the undercarboxylated (GLU13) form of osteocalcin (Figure 3E). This is more severe than what is seen in Osteocalcin ${ }^{+/-}$mice (17). Ctsk-Cre;DTA ${ }^{f l /+}$ transplanted mice also demonstrated a significant reduction in their sperm count and circulating testosterone levels (Figure 3, F and G). Testes, epididymal, and seminal vesicle weights were also reduced, while circulat- ing LH levels were higher in Ctsk-Cre;DTA ${ }^{f l+}$ mice (Figure 3, H-K, and Supplemental Figure 8A). Moreover, expression of the genes encoding the major enzymes required for testosterone biosynthesis (StAR, Cyp11a, Cyp17, 33-HSD) was decreased in Ctsk-Cre;DTA ${ }^{f /+}$ testes, while expression of HSD-17 and Cyp19a was not (Figure $3 \mathrm{~L}$ ). These phenotypic and molecular abnormalities mimic what is seen in Osteocalcin ${ }^{-1-}$ male mice (2), suggesting that osteoclastic bone resorption is necessary for normal male fertility by increasing osteocalcin bioactivity. To add support to this notion, we treated Ctsk-Cre;DTA ${ }^{f l /+}$ transplanted mice with recombinant osteocal$\operatorname{cin}(3 \mathrm{ng} / \mathrm{g}$ of BW, 30 days). At the end of this treatment period, the defects in testes, epididymides, and seminal vesicle weights, sperm count, and circulating testosterone levels observed in CtskCre;DTA ${ }^{f l /+}$ transplanted mice had all been corrected (Supplemental Figure 4, B-D). The detrimental effects of irradiation on spermatogenesis and testis function could certainly explain the lower values of the data presented in Figure 3.

Second, to add credence to the notion that bone resorption is a determinant of the ability of osteocalcin to regulate male fertility, we next studied a gain of function model. Osteoprotegerin (OPG) is a decoy receptor inhibiting the function of the osteoclast differentiation factor RANKL, and both $\mathrm{Opg}^{+/-}$and $\mathrm{Opg}^{-1-}$ mice are osteoporotic because of a vast increase in the number of osteoclasts (19). We noted that there was in $\mathrm{Opg}^{-/-}$mice a massive increase in the undercarboxylated (GLU13), i.e., active form of osteocalcin (Figure 4A). A smaller increase of undercarboxylated osteocalcin was also observed in $\mathrm{Opg}^{+/-}$mice. Consequently, testes, epididymides, and seminal vesicle weights, sperm count, and circulating testosterone levels were all increased in $\mathrm{Opg}^{-1-}$ mice and to a lesser extent in $\mathrm{Opg}^{+/-}$(Figure 4, B-F, and Supplemental Figure 8B), and expression of StAR, Cyp11a, Cyp17, $3 \beta-H S D$ was increased in $\mathrm{OPg}^{+/-}$and $\mathrm{Opg}^{-/-}$testes while expression of HSD-17 and Cyp19a 

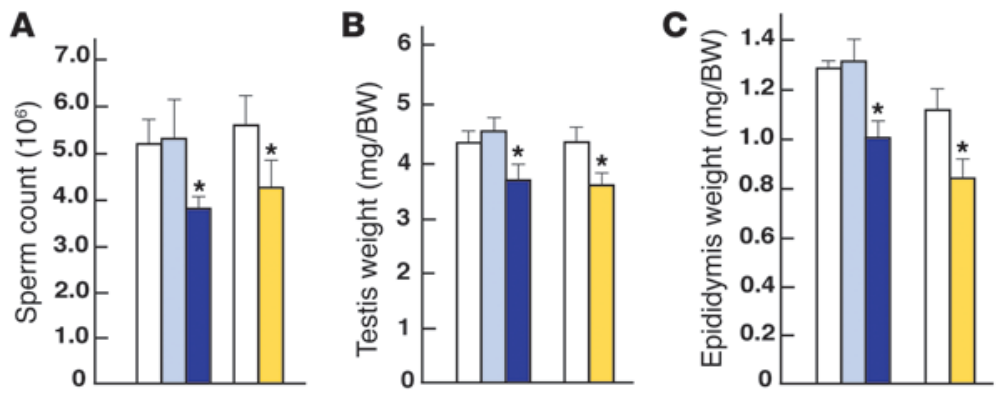

\begin{abstract}
Figure 6
Insulin signaling in osteoblasts promotes male fertility in an osteocalcin-dependent manner. (A) Sperm counts, (B-D) testis, epididymal, and seminal vesicle weights normalized to BW ( $\mathrm{mg} / \mathrm{g}$ of BW); (E) circulating testosterone levels in Ins $R_{o s b^{+/}}(n=5)$, Ins $R_{o s b^{+/-}}$;Osteocalcin ${ }^{+/-}$ $(n=6)$, and InsR $R_{o s b^{+/}}$; Gprc6a $a^{+-}(n=6)$ versus control $(n>8)$ male mice. All analyses presented were performed on nonbreeder mix background (129/Sv: 75\%; C57BL/6J: $25 \%)$ mice. ${ }^{*} P<0.05 ;{ }^{* *} P<0.01$.
\end{abstract}
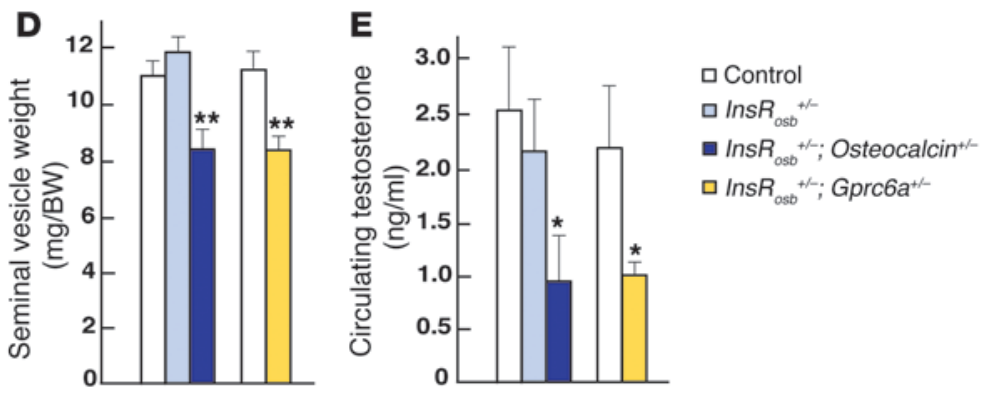

was not (Figure 4G). Finally, circulating LH levels were lower in $\mathrm{Opg}^{+/-}$and $\mathrm{Opg}^{-/-}$compared with WT serum.

Third, to more firmly establish that it is the increase in undercarboxylated osteocalcin that explains the male reproduction phenotype of Opg-deficient mice, we took advantage of the fact that the $O p g$ mutation is dominant and therefore that $\mathrm{Opg}^{+/-}$mice have an increase in bone resorption and in male fertility parameters (Figure 4). Hence, we asked what would be the consequences of deleting 1 allele of Osteocalcin from $\mathrm{Opg}^{+/-}$mice $\left(\mathrm{Opg}^{+/-} ; \mathrm{Osteocalcin}^{+-}\right)$. The circulating levels of the undercarboxylated form of osteocalcin that were high in $\mathrm{Opg}^{+/-}$were normalized in $\mathrm{Opg}^{+/-}$;Osteocalcin ${ }^{+/-}$male mice (Supplemental Figure 5). Taken together, results gathered from the analysis of loss- and gain-of-function models of bone resorption indicate that, in the mouse, bone resorption is a physiological determinant of osteocalcin's regulation of male fertility through its ability to activate osteocalcin.

Insulin signaling in osteoblasts favors testosterone biosynthesis in the mouse. The cardinal role of bone resorption in the regulation of male fertility provided an opportunity to look for additional upstream regulators of osteocalcin reproductive function. Since it is a positive regulator of bone resorption and it promotes reproductive function, we tested here the hypothesis that insulin signaling in osteoblasts might influence testosterone biosynthesis in an osteocalcin-dependent manner.

For that purpose, we analyzed 12-week-old male mice lacking the gene encoding for insulin receptor selectively in osteoblasts $\left(\operatorname{Ins} R_{o s b^{-}}{ }^{--}\right)$ (16). These animals that have lower circulating levels of active osteocalcin demonstrated a decrease in testes size and weight, in epididymides and seminal vesicle weights, in sperm count and circulating testosterone levels (Figure 5, A-F). Moreover, and as is the case in Osteocalcin ${ }^{-1-}$ male mice, expression of StAR, Cyp11a, Cyp17, 33-HSD was decreased in $\mathrm{Ins}_{\text {os }}{ }^{-1-}$ testes, while HSD-17 and Cyp19a expression was not (Figure 5G). Thus the phenotypic, biochemical, and molecular abnormalities of $I n s R_{o s b}{ }^{-1-}$ male mice are indistinguishable from those of Osteocalcin ${ }^{-/-}$male mice (2). These results implied that, as is the case for metabolism, insulin signaling in osteoblasts favors testosterone biosynthesis by increasing osteocalcin bioactivity.
To demonstrate that it is indeed the case, we next generated compound mutant mice lacking 1 allele of InsR in osteoblasts and 1 allele of either Osteocalcin or Gprc6a. As shown in Figure 6, A-E, and Supplemental Figure 8, C and D, whether we looked at testis, epididymides, and seminal vesicle weights or sperm count and circulating levels of testosterone, these compound mutant mice demonstrated abnormalities that were similar to those seen in Ins $R_{\text {osb }}{ }^{-1}$, Osteocalcin ${ }^{-/-}$, or, even more relevantly, in Gprc6a $a^{-/-}$mice $(2,4)$. Taken together, these results point toward the existence of a pancreas-bone-testis axis in the control of male fertility that acts in parallel to the hypothalamus-pituitary-testis axis.

Identification of missense mutation in GPRC6A. In the second part of this study, we addressed another critical aspect of osteocalcin biology, namely, its relevance to humans. To date, the only direct, i.e., genetic evidence that Osteocalcin is a hormone has been obtained in mice. However, the biological importance of osteocalcin would be greatly enhanced if it could be shown to have conserved its function in humans as well.

To address this question, we looked at a patient populations with a well-defined phenotype. The reproductive phenotype of male Osteocalcin ${ }^{-/}$and Gprc6a $a^{-/}$mice is characterized by subfertility in the confined environment of a mouse facility, mediocre spermogram, low circulating testosterone levels, and high circulating LH levels (2). This presentation is similar to that of peripheral testicular insufficiency in humans (20-23). Thus, we performed a genomic analysis of patients harboring similar reproductive abnormalities to Osteocalcin ${ }^{-1}$ mice with the goal of identifying loss-of-function mutation in Osteocalcin or GPRC6A that would explain their clinical presentation. We are aware that GPRC6A may have several ligands in vitro; however, the only one for which there is genetic evidence that it binds to this receptor is osteocalcin.

Out of 1,700 new patients seen in an outpatient clinic for fertility disorders, we obtained DNA samples of 59 male patients aged from 32.2 to 49.4 years who had been diagnosed for infertility with oligospermia (sperm count less than 5 million/ml) and high LH circulating levels, but without a corresponding increase in circulating testosterone levels. We used these 3 parameters as a definition 
A

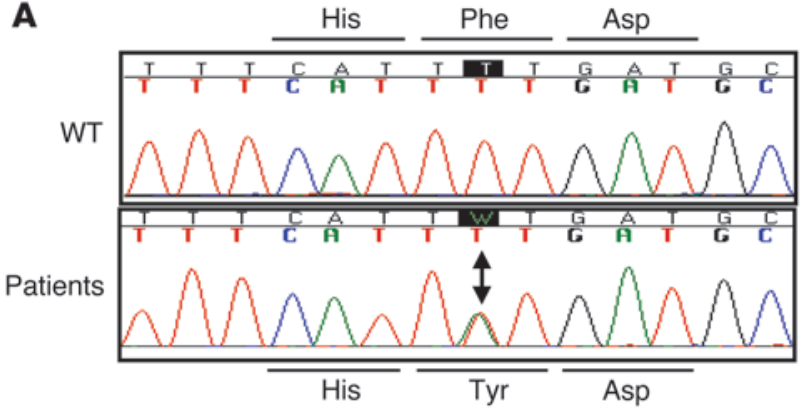

c

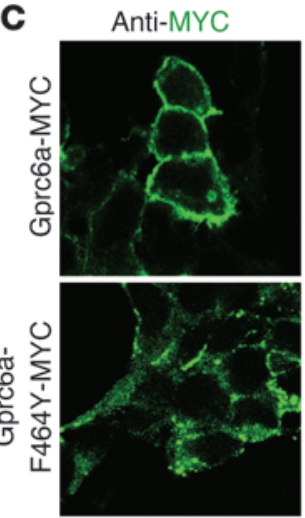

E

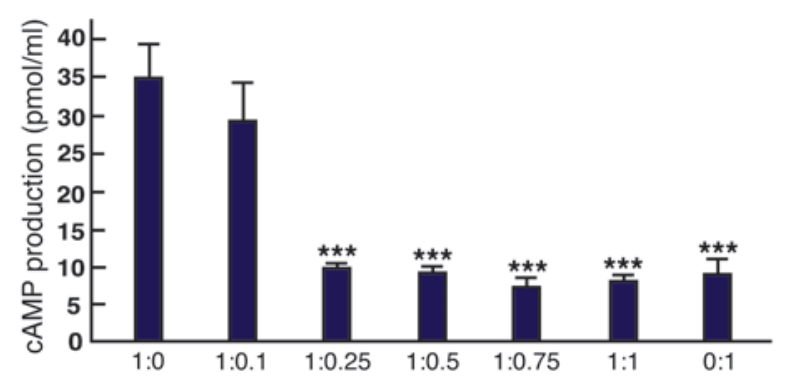

B

GPRC6A (6 exons: 2860pb)

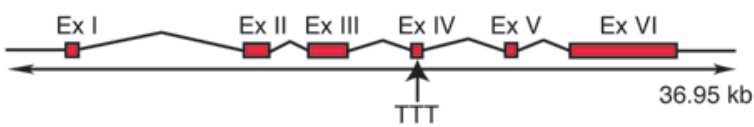

Merge-DAPI
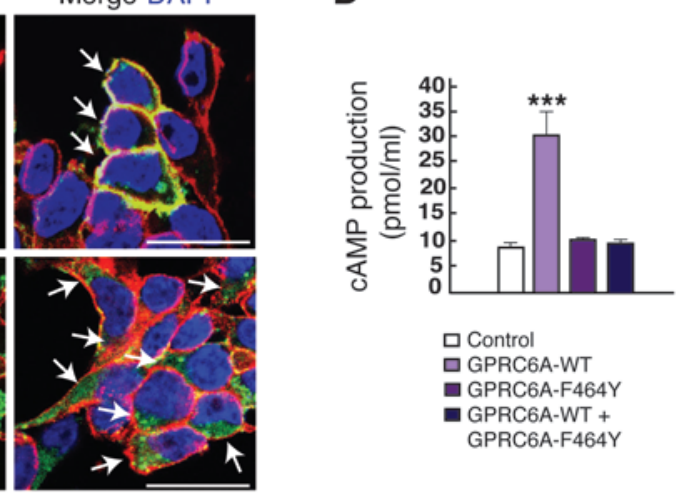

$\square$ Control

口 GPRC6A-WT

GPRC6A-F464Y

- GPRC6A-WT +

GPRC6A-F464Y

$\mathbf{F}$

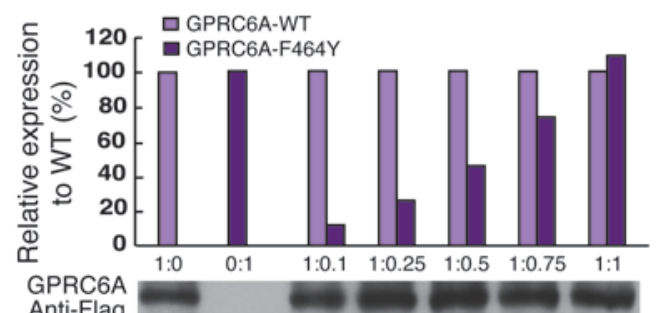

Anti-Flag

Anti-Tubulin

GPRC6A-F464Y

Anti-myc

Anti-Tubulin
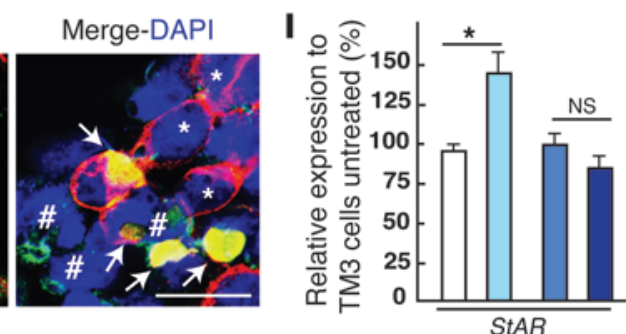

口GPC6A-WT untreated 口GPC6A-WT +

osteocalcin $(1 \mathrm{ng} / \mathrm{ml})$

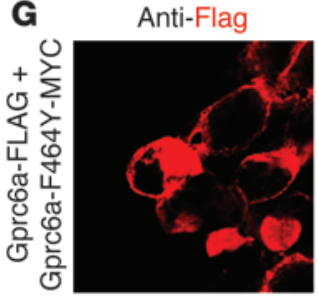

Anti-MYC
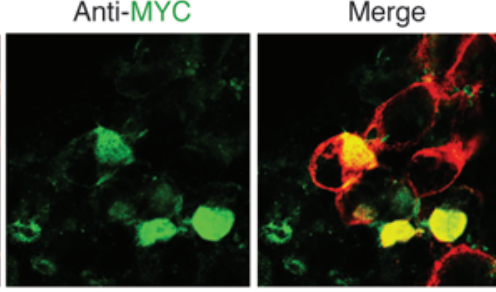

Merge-DAPI
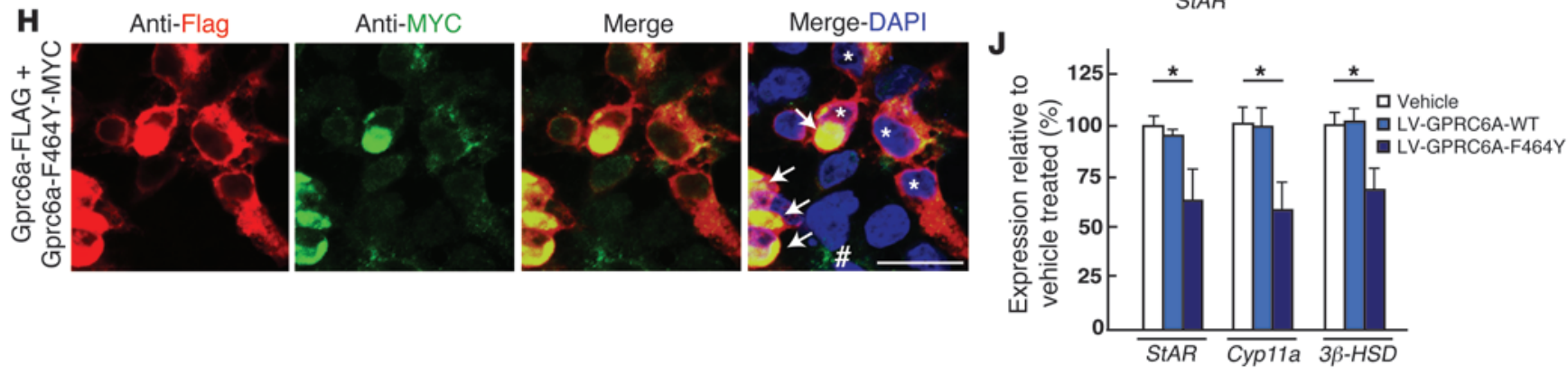


\section{Figure 7}

Identification of an amino acid substitution of GPRC $6 \mathrm{~A}$ associated with decreased fertility in humans. (A) $\mathrm{T} \rightarrow \mathrm{A}$ missense mutation in GPRC6A seen in 2 patients. (B) Localization of the F464Y substitution-mutation in GPRC6A. (C) Immunofluorescence of HEK293T cells transfected with WT (GPRC6A-MYC) or mutant (GPRC6A-MYC-F464Y) GPRC6A. Nuclei are stained with DAPI (blue), MYC-tag in green, and the membrane marker $\alpha 1$ sodium potassium ATPase in red. (D) CAMP production upon osteocalcin stimulation $(3 \mathrm{ng} / \mathrm{ml})$ in HEK293T cells transfected with control (MYC-pcDNA3), WT (GPRC6A-pcDNA3), mutant (GPRC6A-mutant-pcDNA3), or both forms of GPRC6A. (E) cAMP production upon osteocalcin stimulation $(3 \mathrm{ng} / \mathrm{ml})$ in HEK293T cells transfected with WT (GPRC6A-pcDNA3), mutant (GPRC6A-mutantpcDNA3) or both forms of GPRC6A (ratio of 1[WT]: 0.1 [mutant], $1: 0.25,1: 0.5,1: 0.75$, and 1:1). (F) qPCR and Western blot analysis of HEK293T cells cotransfected with WT (GPRC6A-FLAG) and mutant (GPRC6A-MYC-F464Y) GPRC6A at different ratios. (G and H) Immunofluorescence analyses of HEK293T cells cotransfected with WT and mutant GPRC6A at a ratio of $(\mathbf{G})$ 1:1 (WT/mutant) and $(\mathbf{H})$ 1:0.25. FLAG-tag stained in red, MYC-tag in green, and nuclei in blue (DAPI). (I) qPCR analysis of StAR expression in TM3 cells infected with pLenti6.3/V5 lentiviral vectors containing WT GPRC6A or the F464Y mutant and treated with vehicle or osteocalcin $(1 \mathrm{ng} / \mathrm{ml})$. (J) qPCR analysis of StAR, $3 \beta-H S D$, and Cyp11a expression in WT testis injected with vehicle, WT GPRC6A (LV-GPRC6A-WT), or the GPRC6A-F464Y-mutant (LV-GPRC6A-F464Y) ( $n=4$ for each conditions). Scale bar: $10 \mu \mathrm{m}$. ${ }^{\star} P<0.05 ;{ }^{* \star \star} P<0.001$.

of primary testicular failure (20-23). All tested patients had a normal karyotype (14-17). We sequenced all exons of Osteocalcin and GPRC6A, the receptor mediating osteocalcin reproductive function in Leydig cells (2), in these patients. Two patients in this cohort harbored a $\mathrm{T} \rightarrow \mathrm{A}$ transversion in exon 4 (g.117121904A/T), resulting in an amino acid substitution in a transmembrane domain of GPRC6A (F464Y) (Figure 7, A and B, and Supplemental Figure $6 \mathrm{~A})$. Cross-species alignment showed that the F464 position is conserved down to zebra fish (Figure 7, A and B, and Supplemental Figure 6A). Accordingly, the F464Y substitution is considered to be deleterious by all prediction programs tested: Polyphen 2 (probably damaging, score $=1$ ), SIFT (not tolerated), PhD-SNP (disease related, $\mathrm{RI}=6)$, and Panther $(\mathrm{P}$ deleterious $=0.96)$.

Next, we sequenced as controls 278 individuals originating from Senegal and Togo, the countries of origin of the patients with the GPRC6A mutation, all with normal spermogram, and did not find any harboring this mutation. We also sequenced the exon 4 of GPRC6A in 664 additional male controls, who were either African American, of mixed European descent, or Asian and also failed to find this mutation in any of them. Hence, we found this mutation in 2 out of 59 patients (3.39\%) and in none of the 942 controls (Fisher exact test $P=0.036$ vs African controls and $P=0.003$ vs. all controls). We pursued our analysis and also searched public databases for this single nucleotide variant: the F464Y substitution was detected in 52/4352 African-American and 2/8598 (0.02\%) European chromosomes sequenced in the NHLBI GO Exome Sequencing Project, a study whose principal aim is to discover novel genes underlying cardiovascular disorders. These data suggest that $\mathrm{F} 464 \mathrm{Y}$ is a rare allele of GPRC6A.

The first patient was a 45 -year-old man originating from Togo, with no medical records except that indicating an inability to procreate. His parents were deceased; however, we were able to test his sister, who did not harbor the mutation described below (Supplemental Figure 6B). The patient presented a testicular volume decreased by $6 \mathrm{ml}$ for the right side and $5 \mathrm{ml}$ for the left. Semen analysis showed an oligospermia with $2.71 \mathrm{million} / \mathrm{ml}$ (normal $>40$ ), normal vitality with $6 \%$ necrospermia, low bioavailable testosterone levels $(1.4 \mathrm{nmol} / 1$; normal range $=2.3-10.7 \mathrm{nmol} / \mathrm{l})$, elevated LH (12 UI/1; normal range $=0.5-10 \mathrm{UI} / 1)$ and follicle-stimulating hormone (FSH) levels (between 26.7-32.6 UI/1; normal range $=1.3-11.5 \mathrm{UI} / \mathrm{l})$, and decreased inhibin $\mathrm{B}(<15 \mathrm{ng} / \mathrm{l})$ (Table 1). The patient had normal bone mass and bone microarchitecture as assessed by HR-pQCT (Scanco) and normal circulating levels of osteocalcin (Table 1).

The similarity between the clinical presentation of this patient and the phenotype of Osteocalcin ${ }^{-/-}$mice extended further. Indeed, this patient presented a metabolic syndrome characterized by an increase in BMI $\left(31.6 \mathrm{~kg} / \mathrm{m}^{2}\left[\mathrm{Nl}<25 \mathrm{~kg} / \mathrm{m}^{2}\right]\right)$, elevated waist circumference $(107 \mathrm{~cm})$, an increase in adiposity at $28.1 \%$ as assessed by whole-body densitometry, and dyslipidemia as well as glucose intolerance determined by hyperinsulinemia after fasting, a glucose tolerance test, and an insulin tolerance test (Tables 1 and 2). Many of these features are seen in mice lacking Osteocalcin or Gprc6a in all cells (2-5).

The second patient was a 35 year-old man originating from Senegal (Tables 1 and 2, and Supplemental Figure 6C) who sought medical advice after 4 attempts of intracytoplasmic sperm injection failed to result in pregnancy. He has a history of high glucose blood levels that is kept under control by intensive daily exercise and a strict caloric restriction (Tables 1 and 2, and Supplemental Figure 6C). The patient reported a left orchidectomy for cryptorchidism at 8 years of age. Right testicular volume was $18 \mathrm{ml}$. Semen analysis showed an extreme oligospermia with 0.87 million/ml (normal > 40); vitality was decreased with $21 \%$ necrospermia. Relatively low levels of bioavailable testosterone $(4.7 \mathrm{nmol} / 1$; normal range $=2.3-10.7$ $\mathrm{nmol} / \mathrm{l})$ in the face of elevated LH levels $(17 \mathrm{UI} / \mathrm{l}$; normal range $=$ 0.5-10 UI/l) confirmed the diagnosis of primary testicular failure (Table 1). FSH levels were slightly elevated at $7.23 \mathrm{UI} / 1$ (normal range $=1.3-11.5 \mathrm{UI} / \mathrm{l})($ Table 1$)$. To rule out the possibility that his oligospermia and sterility were a direct consequence of his unilateral cryptorchidism, we compared his sperm count to those of the 9 control individuals who also had orchidectomy due to unilateral cryptorchidism. As presented in Supplemental Table 1, the sperm count in these controls was an average of 17.8 million $/ \mathrm{ml}$, i.e., 20-fold higher than in this patient. Physical examination noted the following: weight, $79 \mathrm{~kg}$; height, $1.78 \mathrm{~m}\left(\mathrm{BMI}=25 \mathrm{~kg} / \mathrm{m}^{2}\right)$; and blood pressure, 120/70 mmHg (see Tables 1 and 2).

The F464Y amino acid substitution in GPRC6A acts as a dominant negative mutation. When examined in cell culture, the F464Y substitution-mutation resulted in a loss of function of GPRC6A. First, when HEK293T cells were transfected with this mutated form of GPRC6A, osteocalcin stimulation failed to increase cAMP production as it did in cells transfected with WT GPRC6A (Figure 7). Second, immunofluorescence of HEK293T cells transfected with either a WT or a F464Y mutated MYC-tagged version of GPRC6A showed that this mutation prevented the localization of the receptor to the cell membrane, which instead accumulated in the endoplasmic reticulum (Figure 7C). Osteocalcin also failed to increase cAMP production in cells cotransfected with equal amounts of expression vector for the WT and F464Y mutated forms of GPRC6A (Figure 7D). Western blot analysis confirmed that similar amounts of the WT and F464Y mutated forms of GPRC6A were present in HEK293T cells transfected with each cDNA (Supplemental Figure 7A). 
Table 1

Hormonal and metabolic profiles of the patient presenting an F464Y amino acid substitution of GPRC6A

\begin{tabular}{|c|c|c|c|c|}
\hline & & Patient 1 & Patient 2 & Normal range \\
\hline \multirow[t]{3}{*}{ Bone profile } & Calcium (mmol/l) & 2.3 & 2.2 & $2.12-2.52$ \\
\hline & PTH $(p g / m l)$ & 35 & 40 & $15.0-65.00$ \\
\hline & Osteocalcin (ng/ml) & 14 & 18 & $14.0-42.0$ \\
\hline \multirow[t]{4}{*}{ Metab profile } & $\mathrm{HbA} 1 \mathrm{C}(\%)$ & 6.5 & 5.20 & $4.0-6.0$ \\
\hline & Glycemia (mmol/l) & 5.8 & 4.90 & $4.1-5.8$ \\
\hline & Insulin (mUl/l) & 35.7 & 9.10 & $2.0-25.0$ \\
\hline & C-peptide (nmol/l) & 2.19 & 0.96 & $0.22-0.83$ \\
\hline \multirow[t]{4}{*}{ Sexual hormonal profile } & Free testosterone $(\mathrm{nmol} / \mathrm{l})$ & 1.42 & 4.70 & $2.25-10.7$ \\
\hline & $\mathrm{LH}(\mathrm{UI} / \mathrm{l})$ & 13.10 & 17.00 & $0.5-10.0$ \\
\hline & FSH (UI/I) & 32.60 & 7.23 & $1.3-11.5$ \\
\hline & Estradiol (pmol/l) & 47.00 & 46.70 & \\
\hline \multirow[t]{4}{*}{ Body densitometry } & $\mathrm{BMI}$ & 31.6 & 25.00 & $<25$ \\
\hline & Total mineral mass (BMC) (\%) & 3.06 & 3.6 & \\
\hline & Body fat mass (\%) & 28.07 & 20.20 & $15-20$ \\
\hline & Lean body mass ( $\%)$ & 68.92 & 76.20 & \\
\hline
\end{tabular}

Calcium serum content, parathyroid hormone (PTH) (pg/ml), osteocalcin, metabolic profile HbA1C, glycemia, insulin, C-peptide, serum free testosterone, LH, FSH, estradiol, body densitometry (BMI), total mineral mass, body fat mass, and lean body mass in patients presenting an F464Y amino acid substitution of GPRC6A. Numbers in bold indicate values that are either elevated or decreased compared with normal levels. Metab, metabolic; BMC, bone mineral content.
Further experiments suggested that this F464Y substitution in GPRC6A acts as a dominant negative mutation. Indeed in cotransfection experiments using a fixed amount of the WT form of GPRC6A and increasing amounts of its mutated form, cAMP production was significantly decreased whether cotransfections were performed at a ratio of 1:0.25 (WT/mutated), 1:0.5, 1:0.75, or 1:1 (Figure 7E). Western blot and quantitative PCR verified the different levels of expression of the mutated and WT forms of GPRC6A at the different ratios (Figure 7F). Immunofluorescent staining on transfected HEK293T cells showed that the WT form of GPRC6A never reached the cell membrane when it was cotransfected with the mutated form of GPRC6A (Figure 7G). Instead, it remained localized with the F464Y mutant around the nucleus of cells cotransfected at the ratio of 1:1 (Figure 7G) and 1:0.25 (Figure 7H).

To add further support to the notion that the F464Y mutation in GPRC6A acts as a dominant negative mutation, we transfected WT and the F464Y GPRC6A in TM3 cells. We observed that StAR expression was increased by osteocalcin in TM3 Leydig cells overexpressing WT GPRC6A, but not in TM3 cells overexpressing the F464Y mutant (Figure 7I). Finally, WT mice were injected intratesticularly with a lentivirus expressing either WT GPRC6A or the F464Y mutant. As a control, the contralateral testis of each mouse was injected with the vehicle. Three weeks later, the expression levels of $3 \beta-H S D$, StAR, and Cyp11a were analyzed by qPCR. As shown in Figure 7J, WT testes injected with vehicle or with WT GPRC6A lentivirus demonstrate a level of expression similar to that in these 3 genes, while expression of these genes was significantly reduced in the testes injected with the lentivirus expressing the F464A mutated form of this receptor. This decrease in $3 \beta-H s d$, StAR, and Cyp11a was of the same sever- ity if not more severe than that observed in Gprc6a-deficient testis (2), suggesting that the F464Y mutation acts as a dominant negative in vivo.

Taken together, results of these 3 different cell culture and in vivo assays are consistent with the notion that the F464Y substitution in GPRC6A may be a cause of the primary testicular failure observed in these 2 patients.

\section{Discussion}

This study was initiated with 2 purposes. The first was to identify endocrine regulator(s) of osteocalcin reproductive functions. The second was to use the reproductive function of osteocalcin observed in the mouse as a tool to search for loss-of-function mutations in this hormone or its receptor in humans.

Leydig cells of the testis are responsible for producing testosterone, which in turn determines the development of male reproductive organs, maturation of germinal cells, and generation of estrogen by aromatization. By and large, the main physiological regulator of testosterone production by Leydig cells is the pituitary hormone $\mathrm{LH}(13,24)$. Accordingly, male mice lacking LH or its receptor are sterile, with low levels of circulating testosterone. Given this knowledge, it was unexpected that another hormone would influence testosterone biosynthesis and that this hormone, osteocalcin, was made in bone. Yet, the fact that Osteocalcin ${ }^{-/-}$male mice are subfertile with a marked decrease in circulating testosterone and low sperm count established the biological relevance of this regulation in vivo in the mouse (2).

The fact that osteocalcin injections cannot rescue the sterility of $\mathrm{Lhb}^{-/-}$male mice while it can do so in Osteocalcin-/- mice and the complete rescue of the subfertility of Osteocalcin ${ }^{-1-}$ male mice after hCG injections are not consistent with a model in which osteocalcin would be a mediator of LH in the male mouse. This conten-

\section{Table 2}

Oral glucose tolerance test performed in the patients presenting an F464Y amino acid substitution of GPRC6A

$\begin{array}{lcccccc}\text { OGTT } & \begin{array}{c}\text { Glycemia } \\ \text { mmol/l }\end{array} & \begin{array}{c}\text { TO } \\ \text { Insulin } \\ \text { mUI/l }\end{array} & \begin{array}{c}\text { C-Peptide } \\ \text { nmol/l }\end{array} & \begin{array}{c}\text { Glycemia } \\ \text { mmol/I }\end{array} & \begin{array}{c}\text { Insulin } \\ \text { mUl/l }\end{array} & \begin{array}{c}\text { C-Peptide } \\ \text { nmol/l }\end{array} \\ \text { Patient 1 } & \mathbf{5 . 9 6} & \mathbf{3 8 . 8} & \mathbf{2 . 1 2} & \mathbf{1 1 . 1 2} & \mathbf{7 3 9 . 5} & \mathbf{1 0 . 5 5} \\ \text { Patient 2 } & 4.50 & 5.1 & 0.70 & \mathbf{4 . 0 0} & \mathbf{2 8 . 8} & \mathbf{2 . 1 3} \\ \text { Control } & 5.27 & 0.73 & 10.3 & 3.05 & 12.4 & 1.89\end{array}$

Glycemia $(\mathrm{mmol} / \mathrm{l})$, insulin $(\mathrm{mUl} / \mathrm{l})$ and C-peptide $(\mathrm{nmol} / \mathrm{l})$ were measured in serum of the patient harboring the F464Y substitution of GPRC6A and compared with a control. The blood samples were collected after 12 hours fasting (TO) prior to the test and 2 hours after oral administration of $75 \mathrm{mg}$ of glucose solution to drink. The 2 hours oral glucose tolerance test (OGTT) glucose level should be below $7.8 \mathrm{mmol} / \mathrm{l}$. Levels between this and 11.1 $\mathrm{mmol} / \mathrm{l}$ indicate impaired glucose tolerance. Glucose levels above $11.1 \mathrm{mmol} / \mathrm{l}$ at 2 hours confirms a diagnosis of diabetes. 
tion is further supported by the facts that LH does not regulate Osteocalcin expression, that osteocalcin signaling in Leydig cells does not require $\mathrm{LH}$, and that testosterone inhibits $L b$ expression equally well whether osteocalcin signaling is present or absent in pituitary gland. Taken together, these results dissociate LH-dependent from osteocalcin-dependent regulation of testosterone in male mice and instead suggest the existence of a second endocrine axis including bone and osteocalcin that is necessary for optimal male fertility in vivo in the mouse. They also illustrate the power of mouse genetics in enabling us to understand unexplained but classical clinical presentations. Even though Gprc6a expression in the hypothalamus and pituitary gland is the same in WT and Gprc6a-null mice, we cannot exclude a priori the formal possibility that this receptor may be expressed elsewhere in the brain and that it could, through this putative brain expression, contribute to the regulatory loop described in this study.

Who are the players in this second axis? We show here that bone resorption by osteoclasts through its ability to decarboxylate osteocalcin favors male fertility and that this function is enhanced by insulin signaling in osteoblasts. These results are consistent with the notion that insulin favors reproductive function, but do not exclude that, as shown before, insulin acts through other pathways to favor fertility (25-29). By extension, one can hypothesize that other endocrine or neuronal regulators of bone resorption such as the parathyroid hormone or the sympathetic nervous system may also affect male fertility $(30,31)$. It remains to be determined, however, provided that these regulations exist, whether they occur through osteocalcin or independently of it.

A second legitimate question that has plagued osteocalcin research since this molecule was recognized to be a hormone in rodents has been whether it also has an endocrine function in humans. We used the well-defined reproductive phenotype of the Osteocalcin ${ }^{-1-}$ mice as a tool to explore this question.

When analyzing patients with peripheral testicular insufficiency, we noted that 2 of them were heterozygous for a point mutation in exon 4 of GPRC6A. This missense mutation affected a highly conserved residue, occurred in one of the transmembrane regions of the molecule, and prevented its localization to the cell membrane, therefore resulting in a loss of function of GPRC6A. Three different cell-based assays indicated that this mutation also acts in a dominant negative manner in cells. These results suggest that GPRC6A may be a new susceptibility locus for primary testicular failure in humans, a disease whose cause is often unidentified. The proof that this mutation is sufficient to cause a phenotype of peripheral testicular failure will have to await, however, the generation of mice harboring this single base-pair substitutionmutation. Since the sequencing was restricted to exons, we cannot exclude that mutations in regulatory regions of either Osteocalcin or GPRC6A may be present in other patients suffering from peripheral testicular failure.

We note that both patients harboring this substitution-mutation in GPRC6A originated from Western Africa, shared a history of glucose intolerance, and displayed similar defects in reproductive hormones. It is thus interesting that the F464Y variant was not observed in our ethnicity matched controls with normal spermograms but has a reported frequency of $1.2 \%$ in African-Americans sequenced in the NHLBI exome project, a population that has a higher frequency of cardiovascular and metabolic diseases. Careful phenotypic analysis of individuals carrying the F464Y allele may clarify the spectrum of associated metabolic, cardiovascular, and reproductive defects. Results of this initial foray in the genetic analysis of osteocalcin functions in humans should be viewed as a stepping-stone to performing a more systematic analysis in a larger patient population with primary testicular failure as well as in patients with glucose intolerance or metabolic syndromes.

\section{Methods}

Mouse models. Generation of $\mathrm{Lbb}^{-/}$, Osteocalcin-1-, Gprc6a-/-, Opg ${ }^{-1}$, and Ins $R_{o s} b^{-1 /}$ mice was reported previously $(2,7,16,19,32)$. CtskCre;DTA ${ }^{f /+}$ mice were generated by crossing Ctsk $\mathrm{Cr} / \mathrm{+}^{\mathrm{m}}$ mice, which express Cre recombinase under the control of the osteoclast-specific Cathepsin K locus (16), with $D T A^{f /+}$ mice, which harbor a flox-STOP-flox-DTA gene cassette under the control of the Rosa26 promoter (18). CD 45.2+ fetal liver stem cells isolated from E14.5 control (Ctsk ${ }^{C r e /+}$ and DTA $A^{f /+}$ ) or CtskCre;DTA embryos were transplanted $\left(2 \times 10^{6} / \mathrm{mice}\right)$ via tail-vein injection into 5-week-old CD $45.1^{+}$irradiated WT recipient mice. Genetic backgrounds of mice were as follows: Lbb $^{-/-}$(C57BL/6J: 100\%), Osteocalcin-/- (129/Sv: 100\%), Gprc6a-/- (129/Sv: 100\%), InsR osb $^{-/-}\left(129 / \mathrm{Sv}: 87.5 \%\right.$; C57BL/6J: 12.5\%), InsR osb ${ }^{+/}$;Osteocalcin ${ }^{+/}$ (129/Sv: 75\%; C57BL/6J: 25\%) InsR osb $^{+-}$; $\operatorname{Gprc6a}^{+/-}(129 / \mathrm{Sv:} 75 \%$; C57BL/6J: 25\%), InsR osb $^{+/-}$(129/Sv: 75\%; C57BL/6J: 25\%), Opg ${ }^{-/-}$(C57BL/6J: 100\%), and CtskCre;DTA ${ }^{f /+}$ (C57BL/6J: 100\%). Control littermates were used in all experiments. Mouse genotypes were determined by PCR as previously described $(2,7,16,19,32)$.

Osteocalcin and $h$ CG treatments. Six-week-old mice were treated by i.p. injections for 1 month with hCG (5 U twice a week) or with recombinant osteocalcin ( $3 \mathrm{ng} / \mathrm{g}$ daily) (1) for 1 month.

Human studies. Patients were recruited from the Service de Médecine de la Reproduction, Hôpital Femme-Mère-Enfant (CHU de Lyon) between 2007 and 2011. All patients signed a consent form authorizing genetic research. Patient DNA was stored at $-80^{\circ} \mathrm{C}$ in the DNA bank approved by the National French Biomedicine Agency under accreditation number 06-452 and a local ethics committee. We studied patients who were infertile for more than 24 consecutive months and harboring an elevated $\mathrm{LH}$, over 10 UI/1 (LH IRMA; Beckman Immunotech), without a corresponding increase in bioavailable testosterone and a severe alteration of sperm count of less than 5 million/ejaculation (normal > 40 million). Patients with hypofertility due to Klinefelter syndrome, Y chromosome microdeletion, and hypofertility secondary to chemotherapy were excluded. DNA samples from 59 patients were tested in this study. The 664 DNA controls analyzed were provided by the Service de Médecine de la Reproduction, Hôpital Femme-Mère-Enfant and Ali Gharavi (Columbia University, Department of Medicine and Nephrology).

Mutation screening. Using standard protocols, DNA was preferentially extracted from peripheral blood. Reference sequences of Osteocalcin or GPRC6A were downloaded from the NCBI database (37.1 Build; http:// www.ncbi.nlm.nih.gov/) and the Ensembl genome browser (http://uswest. ensembl.org/index.html). Primers were designed for the 4 exons of human osteocalcin and the 6 coding exons of human GPRC6A. DNA from each sample was amplified using the Platinum PCR SuperMix High Fidelity Amplification Kit (Invitrogen). Amplified PCR products were Sanger sequenced. Sequence analysis was performed using Sequencer 4.8 software. All putative variants were confirmed by bidirectional sequencing.

Murine sperm counts and hormone measurements. Caudal epididymides were minced in $1 \mathrm{ml}$ PBS $1 \mathrm{X}$ and the number of cells released counted after 1 hour. The total sperm count was assessed in the final suspension by using a hemocytometer (33). Circulating levels of testosterone were measured by RIA from Diagnostic Systems Laboratories. Serum levels of carboxylated, undercarboxylated, and total osteocalcin were measured using a triple ELISA assay described previously (34). Circulating levels of LH were measured using a Sandwich enzyme immunoassay performed by 
The Ligands Assay Analysis Core Laboratory of the University of Virginia (Charlottesville, Virginia, USA).

Histology. For immunofluorescence experiments, testes were embedded in optimal cutting temperature compound (Sakura Finetek) and stored at $-80^{\circ} \mathrm{C}$ before the preparation of $3-\mathrm{mm}$ sections. Sections were fixed for 30 minutes in PBS $1 \times / \mathrm{PFA} 4 \%$ at room temperature and overnight in PBS $1 \times / \mathrm{PFA} 1 \%$ at $4^{\circ} \mathrm{C}$. Sections were labeled with primary antibodies against $3 \beta$-HSD, CYP17A (Santa Cruz Biotechnology Inc.), and CYP11A and subsequently probed with secondary Alexa Fluor 488-conjugated goat anti-rabbit (Molecular Probes).

Gene expression studies. RNA was purified from testis using TRIzoL (Invitrogen). RNA isolation, cDNA preparation, and $\mathrm{qPCR}$ analysis was carried out following standard protocols. qPCR analyses were performed using specific quantitative PCR primers from SABiosciences.

Evaluation of rare variants. All variants were evaluated for potential pathogenicity using 4 methodologies. First, public databases (dbSNP, 1000 genomes; http://browser.1000genomes.org/index.html) were consulted to determine whether the variants had previously been detected in reference populations. Coding variants were further cross-referenced with prior publications and mutation databases, such as the Human Gene Mutation Database (HGMD). Missense variants were evaluated for conservation among species using TCoffee and for pathogenic potential using standard prediction programs (i.e., Polyphen, SIFT, PhD-SNP). Synonymous and noncoding variants were evaluated for conservation among other mammalian species (bl2seq feature); synonymous variants were also evaluated for potential aberrant splicing (Human Splice Finder and ESE Finder). The frequencies of selected new variants were determined in healthy controls: 278 Africans, 218 African-Americans, 350 people of mixed European descent, and 96 Asians (total: 664 controls) by direct sequencing. The variant F464Y was recently reported in NCBI as a rare SNP (dbsnp rs14391345).

GPRC6A mutagenesis. The normal GPRC6A-c-myc tagged vector was constructed according to previously described methods (35). The $\mathrm{T} \rightarrow$ A site directed mutagenesis was performed using the Agilent (USA) mutagenesis kit using the following primers: forward, 5'-AAGGATTCATTTCACTATGATGCCCACGGGGA-3'; reverse, 5'-TCCCCGTGGGCATCATAGTGAAATGAATTCCTT-3'.

GPRC6A construction. To construct a flag-tagged GPRC6A construct, the c-myc-tagged GPRC6A plasmid was amplified with the following primers: NotI, 5'-AGCGCGGCCGCGTCGTGGCTATCACTTCTTCCTG; BamHI, 3'-CGGGATCCTCATATACTTGAACTTCTTTTCTGACG. The amplified fragment was digested with NotI and BamHI and subcloned into p3XFLAG-CMV vector.

Lentivirus. Full-length GPRC6A cDNA and its F464Y form were cloned into the pLenti6.3/V5 lentiviral vector (Invitrogen). Lentiviral particles were produced at the Gene Transfer Vector Core of the University of Iowa (Iowa City, Iowa, USA). Mice were injected intratesticularly with $20 \mu \mathrm{l}$ of a saline solution containing or not the lentivirus at $10^{7} \mathrm{TU} / \mathrm{ml}$.

Cell culture, transfection, and immunofluorescence assay. HEK293T cells were cultured at $1.5 \times 10^{5}$ cells/well on coverslips coated with poly-D-lysine in DMEM high-glucose medium complemented with $10 \%$ fetal bovine serum and $1 \%$ penicillin/streptomycin (Sigma-Aldrich) at $37^{\circ} \mathrm{C}$ in a humidified atmosphere at $5 \% \mathrm{CO}_{2}$ overnight. Cells were then transfected with 500 ng of WT GPRC6A-pcDNA or mutant GPRC6A-pcDNA using the Poly- plus jetPRIME Reagent according to the manufacturer's instructions. The eGFP-N1 plasmid was used as a control for transfection efficiency. The immunofluorescence assays were performed 12 hours after transfection. Cells were washed 3 times with PBS, fixed for 10 minutes in $4 \%$ paraformaldehyde at room temperature, permeabilized with $0.01 \%$ Triton X-100 (or not permeabilized as a control), and then incubated with Myc-Tag rabbit mAb (1:200; Cell Signaling), anti- $\alpha 1$ sodium potassium ATPase antibody (1:500, ab7671; Abcam), or monoclonal anti-FLAG M2 (1:500; Sigma-Aldrich). After 24 hours at $4^{\circ} \mathrm{C}$, cells were rinsed with PBS and incubated with Alexa Fluor 488 goat anti-rabbit (1:1000) and/or Alexa Fluor 555 donkey anti-mouse (1:1000) for 2 hours at room temperature. All slides were then mounted with Fluoro-Gel II with DAPI (Electron Microscopy Sciences). Staining was visualized and captured using a Zeiss fluorescent microscope or a Nikon confocal microscope.

cAMP measurement. For cAMP measurement, HEK293T cells were transfected with myc-pcDNA3, GPRC6A-pcDNA3, and GPRC6A mutantpcDNA3, and cotransfected with both GPRC6A and its mutant forms in 6 -cm dishes. Cells were starved for 16 hours (in the presence of $0.1 \%$ BSA) and then preincubated in the presence of $0.5 \mathrm{mM}$ IBMX for 30 minutes and stimulated with $3 \mathrm{ng} / \mathrm{ml}$ of osteocalcin also in the presence of $0.5 \mathrm{mM}$ IBMX for 30 minutes. cAMP concentration was measured with the Parameter cAMP kit (KGE002; R\&D Systems).

Statistics. Statistical analyses were performed using 2-tailed Student's $t$ test. Error bars denote mean \pm SEM. A $P$ value of $<0.05$ was considered to be statistically significant.

Study approval. All experiments involving mice were performed in compliance with federal laws and institutional guidelines and approved by the Institutional Animal Care and Use Committee (IACUC) of Columbia University Animal Care Facility, protocol number: AC-AAAC1910. Patients were recruited for the Service de Médecine de la Reproduction, Hôpital Femme-Mère-Enfant between 2007 and 2011. Patients gave informed consent. The DNA bank was approved by the National French Biomedicine Agency under accreditation number 06-452 and a local ethics committee.

\section{Acknowledgments}

We thank P. Ducy for critical reading of the manuscript. S. Kato for provided the Ctsk-Cre mice, A.N. Economides for providing the $D T A^{f l+}$ mice, E. Clark for providing the $\mathrm{Opg}^{-/-}$mice, Y. Morel and R. Barruet and Z. Zomalheto for collecting patient DNA samples, and R. Mathieux, S. Ailloud, and N. Coutel for performing the blood sampling. We are also grateful to A. Gharavi for providing panels of control DNA samples. This work was supported by grants from the NIH (to G. Karsenty, T.R. Kumar, and M. Ferron), Sanofi Aventis (to G. Karsenty), the Canadian Diabetes Association (to M. Ferron), and the Human Frontier Scientific Program (to F. Oury).

Received for publication July 23, 2012, and accepted in revised form March 15, 2013.

Address correspondence to: Gerard Karsenty, Department of Genetics and Development, 701W 168th Street, Room 1602A HHSC, New York, New York 10032, USA. Phone: 212.305.4011; Fax: 212.923.2090; E-mail: gk2172@columbia.edu.

\footnotetext{
1. Lee NK, et al. Endocrine regulation of energy metabolism by the skeleton. Cell. 2007;130(3):456-469.

2. Oury F, et al. Endocrine regulation of male fertility by the skeleton. Cell. 2011;144(5):796-809.

3. Pi M, Wu Y, Quarles LD. GPRC6A mediates responses to osteocalcin in beta-cells in vitro and pancreas in vivo. J Bone Miner Res. 2011;26(7):1680-1683.

4. Pi M, et al. GPRC6A null mice exhibit osteopenia,
}

feminization and metabolic syndrome. PLoS One. 2008;3(12):e3858.

5. Pi M, Quarles LD. Multiligand specificity and wide tissue expression of GPRC6A reveals new endocrine networks. Endocrinology. 2012;153(5):2062-2069.

6. Ferron M, Hinoi E, Karsenty G, Ducy P. Osteocalcin differentially regulates beta cell and adipocyte gene expression and affects the development of metabolic diseases in wild-type mice. Proc Natl Acad Sci U S A. 2008;105(13):5266-5270.

7. Kumar TR. Functional analysis of LHbeta knockout mice. Mol Cell Endocrinol. 2007;269(1-2):81-84.

8. Ma X, Dong Y, Matzuk MM, Kumar TR. Targeted disruption of luteinizing hormone beta-subunit leads to hypogonadism, defects in gonadal steroidogenesis, and infertility. Proc Natl Acad SciUSA. 
2004;101(49):17294-17299

9. Themmen APN, Huhtaniemi IT. Mutations of gonadotropins and gonadotropin receptors: elucidating the physiology and pathophysiology of pituitarygonadal function. Endocr Rev. 2000;21(5):551-583.

10. Fernandez-Real JM, et al. The relationship of serum osteocalcin concentration to insulin secretion, sensitivity, and disposal with hypocaloric diet and resistance training. J Clin Endocrinol Metab. 2009; 94(1):237-245.

11. Im JA, Yu BP, Jeon JY, Kim SH. Relationship between osteocalcin and glucose metabolism in postmenopausal women. Clin Chim Acta. 2008;396(1-2):66-69.

12. Saleem U, Mosley TJ Jr, Kullo IJ. Serum osteocalcin is associated with measures of insulin resistance, adipokine levels, and the presence of metabolic syndrome. Arterioscler Thromb Vasc Biol. 2010;30(7):1474-1478.

13. Kirmani S, Atkinson EJ, Melton LJ 3rd, Riggs BL, Amin S, Khosla S. Relationship of testosterone and osteocalcin levels during growth. J Bone Miner Res. 2011;26(9):2212-2216.

14. Bousfield GR, Ward DN. Evidence for two folding domains in glycoprotein hormone alpha-subunits. Endocrinology. 1994;135(2):624-635.

15. Pierce JG, Parsons TF. Glycoprotein hormones: structure and function. Annu Rev Biochem. 1981; 50:465-495.

16. Ferron $M$, et al. Insulin signaling in osteoblasts integrates bone remodeling and energy metabolism. Cell. 2010;142(2):296-308.
17. Nakamura T, et al. Estrogen prevents bone loss via estrogen receptor alpha and induction of Fas ligand in osteoclasts. Cell. 2007;130(5):811-823.

18. Yoshikawa Y, et al. Genetic evidence points to an osteocalcin-independent influence of osteoblasts on energy metabolism. J Bone Miner Res. 2011; 26(9):2012-2025.

19. Yun TJ, et al. Osteoprotegerin, a crucial regulator of bone metabolism, also regulates B cell development and function. J Immunol. 2001;166(3):1482-1491.

20. Boisen KA, Main KM, Rajpert-DeMeyts E, Skakkebaek NE. Are male reproductive disorders a common entity? The testicular dysgenesis syndrome. Ann N Y Acad Sci. 2001;948:90-99.

21. Glass AR, Vigersky RA. Testicular reserve of testosterone precursors in primary testicular failure. Fertil Steril. 1982;38(1):92-96.

22. Paduch DA. Testicular cancer and male infertility. Curr Opin Urol. 2006;16(6):419-427.

23. Winters SJ, Troen P. A reexamination of pulsatile luteinizing hormone secretion in primary testicular failure. J Clin Endocrinol Metab. 1983;57(2):432-435.

24. Burns KH, Matzuk MM. Minireview: genetic models for the study of gonadotropin actions. Endocrinology. 2002;143(8):2823-2835.

25. Haffner SM. Sex hormones, obesity, fat distribution, type 2 diabetes and insulin resistance: epidemiological and clinical correlation. Int J Obes Relat Metab Disord. 2000;24(suppl 2):S56-S58.

26. Mah PM, Wittert GA. Obesity and testicular func- tion. Mol Cell Endocrinol. 2010;316(2):180-186.

27. Pasquali R, et al. Effect of obesity and body fat distribution on sex hormones and insulin in men. Metabolism. 1991;40(1):101-104.

28. Pitteloud $\mathrm{N}$, et al. Increasing insulin resistance is associated with a decrease in Leydig cell testosterone secretion in men. J Clin Endocrinol Metab. 2005; 90(5):2636-2641.

29. Pitteloud N, et al. Relationship between testosterone levels, insulin sensitivity, and mitochondrial function in men. Diabetes Care. 2005;28(7):1636-1642.

30. Hinoi E, et al. The sympathetic tone mediates leptin's inhibition of insulin secretion by modulating osteocalcin bioactivity. J Cell Biol. 2008;183(7):1235-1242.

31. Elefteriou F, et al. Leptin regulation of bone resorption by the sympathetic nervous system and CART. Nature. 2005;434(7032):514-520.

32. Ducy P, et al. Increased bone formation in osteocalcin-deficient mice. Nature. 1996;382(6590):448-452.

33. Dakhova O, et al. Dickkopf-like1 regulates postpubertal spermatocyte apoptosis and testosterone production. Endocrinology. 2009;150(1):404-412.

34. Ferron M, Wei J, Yoshizawa T, Ducy P, Karsenty G. An ELISA-based method to quantify osteocalcin carboxylation in mice. Biochem Biophys Res Commun. 2010;397(4):691-696.

35. Kuang D, Yao Y, Lam J, Tsushima RG, Hampson DR. Cloning and characterization of a family $\mathrm{C}$ orphan G-protein coupled receptor. J Neurochem. 2005; 93(2):383-391. 